\title{
Automatic detection of high frequency oscillations during epilepsy surgery predicts seizure outcome
}

Fedele, Tommaso ; van 't Klooster, Maryse ; Burnos, Sergey ; Zweiphenning, Willemiek ; van Klink, Nicole ; Leijten, Frans ; Zijlmans, Maeike ; Sarnthein, Johannes

\begin{abstract}
OBJECTIVE: High frequency oscillations (HFOs) and in particular fast ripples (FRs) in the post-resection electrocorticogram (ECoG) have recently been shown to be highly specific predictors of outcome of epilepsy surgery. FR visual marking is time consuming and prone to observer bias. We validate here a fully automatic HFO detector against seizure outcome. METHODS: Pre-resection ECoG dataset ( $\mathrm{N}=14$ patients) with visually marked HFOs were used to optimize the detector's parameters in the time-frequency domain. The optimized detector was then applied on a larger post-resection ECoG dataset $(\mathrm{N}=54)$ and the output was compared with visual markings and seizure outcome. The analysis was conducted separately for ripples $(80-250 \mathrm{~Hz})$ and FRs $(250-500 \mathrm{~Hz})$. RESULTS: Channel-wise comparison showed a high association between automatic detection and visual marking $(\mathrm{p}<0.001$ for both FRs and ripples). Automatically detected FRs were predictive of clinical outcome with positive predictive value $\mathrm{PPV}=100 \%$ and negative predictive value $\mathrm{NPV}=62 \%$, while for ripples $\mathrm{PPV}=43 \%$ and NPV $=100 \%$. CONCLUSIONS: Our automatic and fully unsupervised detection of HFO events matched the expert observer's performance in both event selection and outcome prediction. SIGNIFICANCE: The detector provides a standardized definition of clinically relevant HFOs, which may spread its use in clinical application.
\end{abstract}

DOI: https://doi.org/10.1016/j.clinph.2016.06.009

Posted at the Zurich Open Repository and Archive, University of Zurich

ZORA URL: https://doi.org/10.5167/uzh-127772

Journal Article

Accepted Version

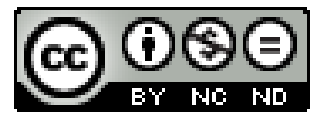

The following work is licensed under a Creative Commons: Attribution-NonCommercial-NoDerivatives 4.0 International (CC BY-NC-ND 4.0) License.

Originally published at:

Fedele, Tommaso; van 't Klooster, Maryse; Burnos, Sergey; Zweiphenning, Willemiek; van Klink, Nicole; Leijten, Frans; Zijlmans, Maeike; Sarnthein, Johannes (2016). Automatic detection of high frequency oscillations during epilepsy surgery predicts seizure outcome. Clinical Neurophysiology, 127(9):3066-3074. DOI: https://doi.org/10.1016/j.clinph.2016.06.009 


\section{Automatic detection of high frequency oscillations during epilepsy surgery predicts seizure outcome}

Tommaso Fedele ${ }^{1}$, Maryse van 't Klooster ${ }^{2}$, Sergey Burnos ${ }^{1,3}$, Willemiek Zweiphenning ${ }^{2}$, Nicole van Klink ${ }^{2}$, Frans Leijten ${ }^{2}$, Maeike Zijlmans ${ }^{2,4}$, Johannes Sarnthein ${ }^{1,5}$

${ }^{1}$ Neurosurgery Department, University Hospital Zurich, Zurich, Switzerland;

${ }^{2}$ Brain Center Rudolf Magnus, Department of Neurology and Neurosurgery, University Medical Center Utrecht, Utrecht, The Netherlands;

${ }^{3}$ Institute of Neuroinformatics, ETH Zurich, Zurich, Switzerland;

${ }^{4}$ SEIN-Stichting Epilepsie Instellingen Nederland, Heemstede, The Netherlands .

${ }^{5}$ Neuroscience Center Zurich, University of Zurich, Zurich, Switzerland

Corresponding author: Tommaso Fedele

Klinik für Neurochirurgie, UniversitätsSpital Zürich

Frauenklinikstrasse 10, CH-8091 Zürich

tel. $+41(0) 442552427$

email: tommaso.fedele@usz.ch

Key words: epilepsy surgery, intraoperative ECoG, high frequency oscillations, fast ripples, automatic detection, seizure outcome 


\section{Abstract}

Objective: High frequency oscillations (HFOs) and in particular fast ripples (FRs) in the postresection electrocorticogram ( $\mathrm{ECoG}$ ) have recently been shown to be highly specific predictors of outcome of epilepsy surgery. FR visual marking is time consuming and is prone to observer bias. We validate here a fully automatic HFO detector against seizure outcome.

Methods: Pre-resection ECoG dataset ( $\mathrm{N}=14$ patients) with visually marked HFOs were used to optimize the detector's parameters in the time-frequency domain. The optimized detector was then applied on a larger post-resection ECoG dataset $(\mathrm{N}=54)$ and the output was compared with visual markings and seizure outcome. The analysis was conducted separately for ripples $(80-250 \mathrm{~Hz})$ and FRs $(250-500 \mathrm{~Hz})$.

Results: Channel-wise comparison showed a high association between automatic detection and visual marking ( $<<0.001$ for both FRs and ripples). Automatically detected FRs were predictive of clinical outcome with positive predictive value PPV $=100 \%$ and negative predictive value NPV $=62 \%$, while for ripples $\mathrm{PPV}=43 \%$ and $\mathrm{NPV}=100 \%$.

Conclusions: Our automatic and fully unsupervised detection of HFO events matched the expert observer's performance in both event selection and outcome prediction.

Significance: The detector provides a standardized definition of clinically relevant HFOs, which may spread its use in clinical application.

\section{Highlights}

- We developed a fully automatic detector for intra-operative high frequency oscillations

- The detector's parameters were validated on large set of intraoperative electrocorticograms

- The detector provides a standardized definition of clinically relevant HFO 


\section{Introduction}

In recent years, interictal High Frequency Oscillations (HFOs, $>80 \mathrm{~Hz}$ ) recorded in epileptic brains have been shown to be a reliable biomarker for the identification of the epileptogenic zone (Jacobs et al. , 2012, Zijlmans et al. , 2012b, Fernandez et al. , 2013). HFOs are classified according to their spectral range in ripples $(80-250 \mathrm{~Hz})$ and fast ripples $(\mathrm{FR}, 250-$ $500 \mathrm{~Hz}$ ) (Bragin et al. , 2010). FR in the intraoperative electrocorticography (ECoG) have been proposed as a predictor of clinical outcome (Wu et al. , 2010, van 't Klooster et al. , 2015b). Both studies provide examples of surgical cases where incomplete resection of electrode locations with HFOs resulted in recurrent seizures. Wu et al. (2010) recorded intraoperatively before resection while van 't Klooster et al. (2015b) recorded intraoperatively after resection.

To date, the identification of HFOs is mainly performed by visual marking or semi-supervised detection (Urrestarazu et al. , 2007, Zijlmans et al. , 2009, Jacobs et al. , 2010, Zelmann et al. , 2010). Automatic detection of HFOs in general, and FRs in particular, suffers from an ensemble of detectability issues, such as the low signal-to-noise ratio and the noisy intraoperative environment. In order to implement the clinical use of HFOs, their value has yet to be confirmed in prospective fashion. The first small clinical trial $(\mathrm{N}=78)$, testing noninferiority of HFOs compared to interictal spikes during surgery, is currently being run (van 't Klooster et al. , 2015a). Evidence for superiority of HFOs over spikes requires an international clinical trial with large numbers of patients, which will need fast, unsupervised and reliable automatic detection of HFOs. Besides reducing the observers' workload, automatic detection would provide a standardized procedure and definition of clinically relevant HFOs.

Several automatic HFO detectors have been developed by different research groups (Staba et al. , 2002, Gardner et al. , 2007, Worrell et al. , 2008, Blanco et al. , 2010, Zelmann et al. , 2010, Dümpelmann et al. , 2012, Birot et al. , 2013, López-Cuevas et al. , 2013, Burnos et al. , 2014, Burnos et al. , 2016b). The general implementation follows a two-stage procedure: a first step aims to identify a reliable threshold that we use to isolate events of interest (EoI), and a second step recognizing HFOs from spurious EoI, e.g. spikes or artifacts, on the basis of a mathematical definition of an HFO. The time-frequency representation appears to be a promising approach to distinguish valid HFOs among EoI, both to detect visually marked events (Wavelet transform, (Birot et al. , 2013)) and to predict clinical outcome (Stockwell transform (Burnos et al. , 2014)). 
We improved a previously described detector (Burnos et al. , 2014) and added a third stage rejecting artifact events occurring synchronously in several channels. The detector, targeting HFOs in intraoperative ECoG recordings, was calibrated on a first dataset of pre-resection recordings with visually marked HFOs (dataset 1, (Zweiphenning et al. , 2015)). After calibration, the detector was applied on a second dataset of post-resection recordings and its output was compared with visual HFO markings and the patient's clinical outcome (dataset 2, van 't Klooster et al. (2015b)). We here evaluate the performance of this completely automatized tool for intraoperative HFO detection, and describe the predictive power of automatically detected post-resection ripples and FRs with respect to seizure outcome.

\section{Methods}

\subsection{Datasets and set-up}

We used two datasets of patients with refractory epilepsy who underwent surgery with intraoperative ECoG (sampling rate $2048 \mathrm{~Hz}$ ) at the University Medical Center Utrecht (UMCU) between 2008 and 2012. These datasets were collected conform the guidelines of the institutional ethical committee of the UMCU.

Dataset 1 consisted of 28 intraoperative pre-resection one minute recordings from 14 patients, two recordings from each patient (Zweiphenning et al. , 2015). The first minute will be called training set and the second minute test set. Dataset 2 consisted of one minute of intraoperative post-resection recordings from 54 patients in which resection sites and clinical outcome had been carefully documented (van 't Klooster et al. , 2015b). The post-resection recordings of the 14 patients with pre-resection recordings in dataset 1 were included in dataset 2 .

ECoG was collected using $4 \times 5$ or $4 \times 8$ electrode grids and $1 \times 6$ or $1 \times 8$ electrode strips (Ad-Tech, Racine, WI) placed directly on the cortex. Platinum electrodes with $4.2 \mathrm{~mm}^{2}$ contact surface, embedded in silicone, and $1 \mathrm{~cm}$ inter-electrode distances were used. Recordings were made with a 64-channel EEG system (MicroMed, Veneto, Italy) at $2048 \mathrm{~Hz}$ sampling rate with an anti-aliasing filter at $538 \mathrm{~Hz}$. Data were analyzed in a bipolar montage along the length of the grid. General anesthesia was induced and maintained using a propofol infusion pump. Propofol was tapered during ECoG registration until a continuous ECoG background pattern was achieved. 
The patients were interviewed in the clinic (UMCU or elsewhere) at the regular follow-up intervals Postsurgical seizure outcome was classified according to Engel's score, dichotomized into good (Engel 1) and poor (Engel $\geq 2$ ) outcome. Follow-up was $>1$ year

\subsection{Visual Analysis}

As stated in the previous publication (van 't Klooster et al., 2015), visual marking of HFO was performed semi-automatically by validating the HFOs identified by the Montreal Neurological Institute detector (MNI detector, Zelmann et al., 2010) adapted for intraoperative ECoG (van Klink et al. , 2014). HFOs were detected if the energy of the signal was larger than baseline during a certain period with a minimum of 4 oscillations. The MNI detector has a high false positive rate, which requires visual validation.

Data were visually inspected in Stellate Harmonie Reviewer (v7.0, Montreal, QC, Canada). ECoG was high-pass filtered using a finite impulse response (FIR) filter $>80 \mathrm{~Hz}$ for ripples and $>250 \mathrm{~Hz}$ for FRs. Split screen allowed to simultaneously visualize ripples (gain 5 $\mu \mathrm{V} / \mathrm{mm}$ ) and FRs (gain $1 \mu \mathrm{V} / \mathrm{mm}$ ) with time interval of 0.4 seconds/page (Jacobs et al. , 2010). Events were visually discarded if it did not fit with the requirements of having at least four oscillations and clearly standing out from the baseline or if it was an artefact. In dataset 1 (pre-resection recordings, $\mathrm{N}=14$ patients) the visual marking was performed in consensus by two reviewers. For dataset 2 (post-resection recordings, $\mathrm{N}=54$ patients), the procedure has been described in detail in (van 't Klooster et al., 2015). The two expert observers were not independent in their visual marking but reached consensus for each event already during the marking process with a $100 \%$ agreement.

\subsection{Automatic Detection}

The automatic detector consists of three stages, which are described in detail below. The analysis was conducted separately for ripples $(80-250 \mathrm{~Hz})$ and FRs $(250-500 \mathrm{~Hz})$. The signal was filtered for the ripple and FR frequency bands by a FIR equiripple filter. For the ripple range filter parameters were set to band-pass $80-240 \mathrm{~Hz}$, with a stopband of $70 \mathrm{~Hz}$ and 250 $\mathrm{Hz}$. For the FR range the band-pass filter was set at $250-490 \mathrm{~Hz}$, with a stopband of $240 \mathrm{~Hz}$ and $500 \mathrm{~Hz}$. In both cases the stopband attenuation was set to $60 \mathrm{~dB}$.

\subsubsection{Stage I: Baseline detection}

We defined as baseline those segments of artifact-free ECoG without oscillations. The baseline was identified by time-frequency resolved entropy in the frequency band of interest, 
similarly to what was previously reported for wavelet entropy (Rosso et al. , 2001, Zelmann et al. , 2010). As entropy kernel, we introduced the Stockwell-transform (Stockwell et al. , 1996). The maximum theoretical Stockwell Entropy ( $\left.\mathrm{SE}_{\max }\right)$ is obtained for white noise. Intervals with sufficiently high Stockwell entropy band, were considered good candidates for the baseline (i.e. no oscillation present). We defined the threshold for baseline entropy BLthr $\mathrm{X}$ $\mathrm{SE}_{\max }$, with $\mathrm{BL}_{\text {thr }}$ being the parameter setting the entropy threshold. The high entropy samples (entropy $>\mathrm{BL}_{\text {thr }} \mathrm{X} \mathrm{SE}_{\max }$ ) were sorted, and their amplitude envelope in the spectral band of interest was used to build the cumulative distribution of the baseline amplitude envelope. The amplitude threshold was set at the cumulative amplitude threshold parameter ( $\left.\mathrm{CDF}_{\text {thr }}\right)$. An EoI was marked when the envelope of the band-passed signal was above the threshold value for a minimum time thr. EoI with an inter-event interval lower than $10 \mathrm{~ms}$ were merged into one EoI. Parameters, $\mathrm{BL}_{\mathrm{thr}}, \mathrm{CDF}_{\mathrm{thr}}$, and thr were optimized on dataset 1 to maximize the detection of visually marked events. The detector with fixed optimized parameters was then applied on dataset 2 .

\subsubsection{Stage II: HFO validation by Stockwell transform}

In the second stage, we distinguished HFOs related EoI from spurious EoI. We assume that HFOs provide a distinct signature in the time-frequency domain, appearing as an isolated energy contribution superimposed on the background activity (Benar et al. , 2010, Crepon et al. , 2010, Birot et al. , 2013). In order to detect such contribution, we computed the Stockwell transform in the period of $0.5 \mathrm{~s}$ preceding and following the boundaries of the EoI. The computing time for time series of $1 \mathrm{~s}$ at $2048 \mathrm{~Hz}$ sampling frequency is comparable to the short-time Fourier transform (<10 ms, with i7-4770 Processor@3.40 GHz). An EoI was defined as HFO if it exhibited a high frequency peak, which was isolated from low frequency activity by a trough in the power spectrum (Burnos et al. , 2014). An illustrative example combining the co-occurrence of a ripple and a FR is shown in Figure 1.

For each EoI, the maximum peak amplitude $\left(\mathrm{HF}_{\text {peak }}\right)$ at frequency $\mathrm{HF}_{\text {freq }}$ is identified in the spectral range of interest (Figure 1E). We defined the trough as the minimum value $\mathrm{LF}_{\text {trough }}$ in the range between the $\mathrm{HF}_{\text {freq }}$ and a lower frequency bound ( $40 \mathrm{~Hz}$ for $\mathrm{R}, 80 \mathrm{~Hz}$ for FR). An EoI is classified as a HFO when the condition $\left(\mathrm{HF}_{\text {freq }}\right)>\left(\mathrm{LF}_{\text {trough }}\right)$ is satisfied for the averaged power spectrum. EoIs with a peak to peak amplitude $>500 \mu \mathrm{V}$ in the ripple range or $>80 \mu \mathrm{V}$ in the FR range were rejected as artifacts. Stage II was adapted from (Burnos et al. , 2014).

\subsubsection{Stage III: Artifact rejection by multichannel information}


As a final step, we used multichannel information to further screen the presence of artifacts in the detected HFOs resulting from stage II. Since HFOs are extremely localized in cortex (von Ellenrieder et al. , 2014, Burnos et al. , 2016a), it is not expected to find HFOs over a large area. For each HFO we calculated the correlation across channels. If the detected HFO exhibited a correlation larger than 0.8 with the signal in channels at a distance $>2 \mathrm{~cm}$ for ripples and $>1 \mathrm{~cm}$ for $\mathrm{FR}$, it was rejected as an artifact.

\subsection{Optimization of baseline detection}

For each patient, we used the two separate minutes (training and test set) of dataset 1 to optimize the detector in the parameter space $\mathrm{BL}_{\mathrm{thr}}, \mathrm{CDF}_{\mathrm{thr}}$, and $\mathrm{t}_{\mathrm{THR}}$. BLthr and $\mathrm{CDF}_{\mathrm{thr}}$ ranged between 0.8 - 0.999 and tTHR between $5-35 \mathrm{~ms}$, for both ripples and FRs. The event-wise performance was evaluated for training and test set separately and quantified for each channel in a Receiver Operating Characteristic (ROC, Table 1). Automatically detected events were rated against visually marked events and classified as true positives (TP), true negatives (TN), false positives (FP), and false negatives (FN). AUC, Sensitivity and Specificity were computed for each input setting. The critical aspect of every detection problem is to define an acceptable balance between TP and FP. We favored a robust low false detection at the expense of minor improvements in TP rates. This choice is explained in Figure 2.

We identified the optimal parameter set by looking at a stable performance in the ROC space across the two minutes for all 14 patients. The final output of the algorithm calibration was, for ripples, $\mathrm{BL}_{\mathrm{thr}}=0.85, \mathrm{CDF}_{\mathrm{thr}}=0.99, \mathrm{t}_{\mathrm{THR}}=20 \mathrm{~ms}$, while for $\mathrm{FR} \mathrm{BL}_{\mathrm{thr}}=0.85, \mathrm{CDF}_{\mathrm{thr}}=0.99$, $\mathrm{t}_{\mathrm{THR}}=10 \mathrm{~ms}$. The ROC for different input settings for the test set is provided as Supplementary Material, Table 1S.

\subsection{Application of the detector on post-resection recordings}

After calibration the optimized detector was applied to dataset 2. The performance of the automatic detection in ripple and FR bands was compared to visual marking.

\subsubsection{Event-wise and channel-wise comparison of HFO detection}

We compared automatically detected and visually marked HFOs at the level of the single event and in terms of distribution across channels. Event-wise comparison for both ripples 
and FRs was based on TPR and FPR. For the channel-wise comparison, the level of association of the spatial distribution of visually marked and automatically detected HFO events was evaluated by $\chi^{2}$ test. A p-value $<0.05$ was considered significant.

\subsubsection{Prediction of clinical outcome}

The output of the HFO automatic detection and visual marking of the post-resection ECoG in dataset 2 was compared to the seizure outcome in each patient. For each patient we calculated the total number of events per channel (number of events), the number of channels with events, and the mean event rate per minute over all channels with events (mean number of events/minute/channels with events). Only channels with an event rate of $>1$ event per minute were considered for further analysis.

We tested for a significant difference in the distribution of mean event rate per minute for ripples and FRs in patients with poor outcome (Engel Ib - IV, recurrent seizures) and good outcome (Engel Ia, seizure-freedom) with the Mann-Whitney U test.

We determined the prognostic value of occurrence of the post-resection HFOs for outcome by calculating the positive predictive value (PPV) and the negative predictive value (NPV) as defined in Table 2 . We compared results for the automatically detected and visually marked HFOs.

The $95 \%$ confidence interval (CI) was calculated on the basis of the binomial distribution. All analysis was performed in MATLAB ${ }^{\circ}$ (www.mathworks.com).

\section{Results}

\subsection{Examples of automatic detections}

In Figure 3 we provide an example of recording setup and analysis workflow from patient 33. Intraoperative post-resection ECoG recordings were collected with subdural grids (Figure 3AB). The ECoG was filtered in ripple and FR band (Figure 3C). The rates of visually marked and automatically detected HFOs were compared (Figure 3D). The automatic detector gave the same spatial distribution as the visual marking for both ripples and FRs.

Figure 4 shows three salient examples of FRs events to compare automatic detection and visual marking in relation to the patient's seizure outcome. Both analyses agreed on the event shown in Figure 4A, therefore classified as a TP. The FR fulfills all criteria for automatic detection as it stands out from the baseline in the filtered trace, and the contribution in the 
$300-400 \mathrm{~Hz}$ range is clearly visible both in the time frequency plane and as a distinct peak in the instantaneous power spectrum. The same criteria were fulfilled for the event in Figure 4B, which was, however, not marked visually, therefore classified as a FP. The event in Figure 4C was marked visually, but not detected automatically (FN). The event duration 6 ms (blue bar) was too short to be accepted as an EoI. Furthermore, there was no distinct contribution in the instantaneous power spectrum.

The first two events occurred in patients with post-resection recurrent seizures, while the third patient was seizure free.

\subsection{Event-wise and channel-wise comparison of HFO detection}

We first compared automatic detection and visual marking on the single event level to quantify at which level the detector emulates an expert observer. In the FR band, comparison gave $\mathrm{TP}_{\mathrm{FR}}=41$ events, $\mathrm{FP}_{\mathrm{FR}}=35$ events, and $\mathrm{FN} F \mathrm{FR}=372$ events. In the 42 patients without FR in visual marking, we counted the non-detection of $\mathrm{FR}$ as $\mathrm{TPR}_{\mathrm{FR}}=1$ and $\mathrm{FPR}_{\mathrm{FR}}=0$. Averaging the event statistics across patients yielded $\mathrm{TPR}_{\mathrm{FR}}=84 \% \pm 1 \%$ and $\mathrm{FPR} F \mathrm{FR}<0.2 \% \pm$ $0.1 \%$. The corresponding analysis in the ripple band yielded $\mathrm{TPR}_{\mathrm{R}}=15 \% \pm 25 \%$ and $\mathrm{FPR}_{\mathrm{R}}=$ $2 \% \pm 4 \%$.

We then compared visual marking and automatic detection on the single channel level. While absolute numbers differ, the spatial distribution of ripples and FR is preserved across channels (Figure 3D). When comparing all channels, we found $\mathrm{TN}=1137$ channels, $\mathrm{FN}=195$ channels, $\mathrm{FP}=14$ channels, and $\mathrm{TP}=15$ channels $\left(\chi^{2}(1, \mathrm{~N}=1361)=29.91, \mathrm{p}<0.001\right)$. In two patients there was partial agreement, and in 44/54 patients visual marking and automatic detection agreed completely in their delineation of the FR area (in total 85\% CI [68\%-90\%]).

The corresponding analysis in the ripple band yielded $\mathrm{TN}=359, \mathrm{FN}=499, \mathrm{FP}=152$, and $\mathrm{TP}=$ $351\left(\chi^{2}(1, \mathrm{~N}=1361)=18.27 \mathrm{p}<0.001\right)$. Partial agreement between automatic and visual analysis was found in 51/54 patients (94\% CI [84\% 99\%]) and disagreement in three patients, all of them with good outcome.

\subsection{Automatically detected HFOs and clinical outcome}

Dataset 2 consisted of 24 patients with poor outcome and 30 with good outcome. The raw and thresholded event rates and mean event rate can be found in supplementary table S2 Postresection FRs (channels with $>1 / \mathrm{min}$ ) were automatically detected in 6 out of 54 patients, who all had post-resection recurrent seizures. This is partially overlapping with the visually marked FRs in 12 patients ( 9 with recurrent seizures and 3 seizure free). Compared to the 
visual markings, automatically detected FRs were found in one additional patient (pt 23) with poor outcome and were not found in seven patients, of whom four had a poor outcome (pt 7, $27,41,44)$ and three a good outcome (pt $8,13,24)$. So $5 / 6$ patients with automatically detected FRs also had visually detected FRs.

Automatic detection led to a PPV of $100 \%(6 / 6$, CI [54\% 100\%]) and a NPV of $62 \%(30 / 48$, CI [47\% 76\%]) vs. a PPV of 75\% (9/12, CI [43\% 94\%]) and NPV 64\% (27/42, CI [48\% $78 \%$ ]) for the visual markings. In eight patients visual markings disagreed with the automatic detections (Figure 5). In seven of these eight patients (patient 7, 8, 13, 24, 27, 41, 44 in Figure 5), 395 FR were marked visually but not automatically. Of those, 247 (62 \%) FR had duration $<10 \mathrm{~ms}$ and were rejected in stage I; 32 (8\%) FR did not have a prominent peak in the high frequency spectrum and were rejected in stage II; 114 (29\%) FR presented high correlation across channels and were then rejected in stage III; two $(<2 \%)$ visually marked FR in patient 13 were thresholded because in these channels the condition FR $>1$ was not met. In patient 23 the automatic detector added $23 \mathrm{FR}$ which had not been visually marked. The mean FR rate for automatic detection was 0.83 per minute for patients with poor outcome and 0 for patients with good outcome (Mann-Whitney $U, p=0.004$, patients without events are included), while for visual markings this was 3.9 per minute for patients with poor outcome and 0.23 per minute for patients with good outcome (Mann-Whitney $U, p=0.018$, patients without events included, Figure 5, Table S2).

We automatically detected ripples in 52 patients, 24 with poor and 28 with good outcome. The distributions of the mean ripple rate for patients with poor and good outcome were not significantly different. In two patients with good outcome we did not find any ripple. This corresponded to a PPV of $43 \%(24 / 52$, CI [32\% 61\%]) and a NPV of $100 \%$ (2/2, CI [15\% $100 \%]$ ). These values are in the same range as what was reported for the visual markings (van 't Klooster et al., 2015b).

The agreement of automatic HFO detection and visual marking was high on the patient level, both for ripples and FRs. Patients with a high FR rate in visual marking were also identified by automatic FR detection. Except for patient 54, the number of automatically detected events, and thus the event rate, was lower than the number of visually marked events.

\section{Discussion}


In this study we propose the design, calibration and validation of an unsupervised tool that is capable of detecting ripples and especially FRs, which are predictive of seizure outcome. Channel-wise comparison showed a high association between automatic detection and visual marking ( $<<0.001$ for both FRs and ripples). Automatically detected FRs predicted clinical outcome (PPV of $100 \%$, NPV of $62 \%$ ). The automatic detection matched the expert observer's visual marking, and provided high predictive power of the seizure outcome. To our knowledge (Höller et al. , 2015) this is the first study where automatic detection was rated against outcome and thereby provides a standardized definition of a clinically relevant HFO.

\subsection{Comparison to the literature}

As a main advantage, automatic detection is reproducible and provides a standardized HFOs rating, while visual marking depends on the observer, and the definition of events may evolve over time. Previous studies have implemented and tested various automatic approaches by considering the visual marking in pre-resection data as the ground truth (Staba et al. , 2002, Gardner et al. , 2007, Zelmann et al. , 2010, Dümpelmann et al. , 2012, von Ellenrieder et al. , 2012). As a difference to previous studies, we divided our data into a training and test set: we used pre- resection data of 14 patients to train and test the detector, and then applied the optimized algorithm to a larger set of post-resection data of 54 patients. Thanks to this machine learning approach, our algorithm should be generalizable to datasets from other centers and scalable to different recording setups.

A benchmarking of four detectors concluded that they suffer from overdetection of HFOs in comparison to visual markings (Zelmann et al. , 2012). In contrast, our detector is more specific than visual marking, which resulted in a low number of false positive and a high number of true negative channels. Still, the high sensitivity towards events was in good agreement with the expert visual observer $(\mathrm{TPRFR}=84 \%)$. This was reflected in the significant channel-wise association between automatic and visual HFO ratings. As major difference with previous studies, we evaluated our detector in terms of predictive power of the seizure outcome, which represents the ultimate goal in optimizing the definition of an HFO. In our detector, the baseline detection is based on oscillatory activity quantified by Stockwell entropy. Strengths of this approach are that it is fast in comparison to wavelet entropy (Zelmann et al. , 2012), and independent of signal amplitude, unlike energy based approaches (Staba et al. , 2002, Gardner et al. , 2007, von Ellenrieder et al. , 2012, Burnos et al. , 2014). While energy based approaches assume that HFOs and other non stationarities do not 
influence significantly the estimation of the backgorund noise level, entropy based methods help isolate time segments carrying the statistical description of the baseline dynamics (Zelmann et al. , 2010, Birot et al. , 2013). However, in recordings lasting more than few minutes, the baseline thresholds can cause problems when the noise in the signal is nonstationary, a common weakness of automatic HFO detection algorithms (Zelmann et al. , 2010).

HFO detectors can be subdivided in time-based (Staba et al. , 2002, Gardner et al. , 2007, Worrell et al. , 2008, Blanco et al. , 2010, Zelmann et al. , 2010, Dümpelmann et al. , 2012, López-Cuevas et al. , 2013) and time-frequency algorithms (Birot et al. , 2013, Burnos et al. , 2014, Burnos et al. , 2016b). In the second step of our detector, we analyze individual blobs in time-frequency domain by disentangling HFOs from broadband artifacts by the duration of the event in the instantaneous power spectra. This new extraction method is an elegant alternative for classification of time-frequency plots.

Another strength of our detector is the use of multi-channel information to identify and reject artifacts and thereby reduce false positive detections. This is a unique approach that simulates visual inspection by expert reviewer, who always will incorporate the other ECoG channels while marking or rejecting a specific event. One can question whether considering visual markings as gold standard is desired. We, therefore, also used comparison to post-resection outcome as a validation of our detector. Events in post-resection ECoG data are the only actual indicators of seizure outcome (van 't Klooster et al. , 2015b).

General limitations of the intraoperative recordings used in this study are the unknown influence of anesthetics on number of HFOs (Zijlmans et al. , 2012a), the influence of the spatial sampling of the macro electrodes (Chatillon et al. , 2013), and the relative small population size in relation to the high number of patients with poor outcome who did not show residual FRs (van 't Klooster et al. , 2015b). The latter may also be related to the relatively short epochs of ECoG analyzed.

\subsection{Clinical implications and future perspectives}

Clinical use of HFOs during epilepsy surgery is hampered by time-consuming analysis. We proposed an unsupervised HFO detector based on the Stockwell transform, which enables fast and accurate detection of HFOs calculation of 1 minute ECoG epochs (e.g. 20 channels, 2 $\mathrm{kHz}$ sampling frequency, 1 minute recording requires 2 minutes of computing time). This is fast enough to facilitate real-time application in the setting of the surgery theatre. 
The detection algorithm easily adapts to differences in recording setup defined by electrode size or spacing (Châtillon et al. , 2013) and electronic noise of the amplifier (Scheer et al. , 2006, Waterstraat et al. , 2015).

For clinical implementation it is also deemed crucial to define a clinical threshold in HFO rate to increase the reliability of the HFO generating areas that need to be removed in order to achieve seizure freedom (Höller et al. , 2015). The question is how can we define such a clinical threshold? Possible strategies for thresholding are the half maximum (Burnos et al. , 2014) standard deviation (Höller et al. , 2015), and post-hoc visual validation (Jacobs et al. , 2010). Prediction of clinical outcome was best for a threshold of $>1$ events per channel per minute. A higher threshold did not affect PPV while it considerably reduced the number of FR events and NPV. Such a clinical threshold might also suit the surgeon better, who is more likely to adjust the resection strategy if $>1$ events are found in one channel to be resected. This way, in $85 \%$ of the patients both visual marking and automatic detection yielded the same HFO area, on which recommendations to the surgeon would be based.

As further validation is required, we envision a detector adapted and applied on different types of datasets from different epilepsy centers, which would result in a generally available automatic procedure and could provide a new standard. It would be beneficial to implement this general detector into clinical EEG software, so it can be used by surgical teams worldwide and experiences can be exchanged. Ideally more data should be collected, preferably prospectively and in randomized controlled trial setting (van 't Klooster et al. , 2015a).

\section{Conclusions}

The automatic and fully unsupervised detection of HFO events matched the expert observer's performance in both event selection and outcome prediction. This algorithm provides a standardized definition of clinically relevant HFOs, and therefore a feasible method for reliably intraoperative HFO detection to help the surgeon guide the resection.

\section{Conflict of Interest Statement}

None of the authors have potential conflicts of interest to be disclosed.. 


\section{Acknowledgements}

We thank Rina Zelman for supporting the visual marking with her detector (Zelmann et al. , 2010). T. Fedele is funded by the Swiss National Science Foundation (SNSF 320030_156029). S. Burnos is funded by the Vontobel Stiftung. M. van 't Klooster is funded by the Dutch Epilepsy Foundation fund number 2012-04. W. Zweiphenning is funded by UMC Utrecht Alexandre Suerman Stipendium. N. van Klink is funded by the Dutch Brain Foundation fund number 2013-139 and the Dutch Epilepsy Foundation fund number 15-09. M. Zijlmans is funded by the Rudolf Magnus Institute Talent Fellowship and ZonMW Veni 91615149. 


\section{References}

Benar CG, Chauviere L, Bartolomei F, Wendling F. Pitfalls of high-pass filtering for detecting epileptic oscillations: a technical note on "false" ripples. Clin Neurophysiol 2010;121:301-10.

Birot G, Kachenoura A, Albera L, Bénar C, Wendling F. Automatic detection of fast ripples. J Neurosci Methods 2013;213:236-49.

Blanco JA, Stead M, Krieger A, Viventi J, Marsh WR, Lee KH, et al. Unsupervised classification of high-frequency oscillations in human neocortical epilepsy and control patients. J Neurophysiol 2010;104:2900-12.

Bragin A, Engel J, Jr., Staba RJ. High-frequency oscillations in epileptic brain. Curr Opin Neurol 2010;23:151-6.

Burnos S, Fedele T, Schmid O, Krayenbühl N, Sarnthein J. Detectability of the somatosensory evoked high frequency oscillation (HFO) co-recorded by scalp EEG and ECoG under propofol. Neuroimage Clin 2015;10:318-25.

Burnos S, Frauscher B, Zelmann R, Haegelen C, Sarnthein J, Gotman J. The morphology of high frequency oscillations (HFO) does not improve delineating the epileptogenic zone. Clin Neurophysiol 2016;127:2140-8.

Burnos S, Hilfiker P, Surucu O, Scholkmann F, Krayenbuhl N, Grunwald T, et al. Human intracranial high frequency oscillations (HFOs) detected by automatic time-frequency analysis. PLoS One. 2014;9:e94381

Chatillon CE, Zelmann R, Hall JA, Olivier A, Dubeau F, Gotman J. Influence of contact size on the detection of HFOs in human intracerebral EEG recordings. Clin Neurophysiol 2013;124:1541-6.

Crepon B, Navarro V, Hasboun D, Clemenceau S, Martinerie J, Baulac M, et al. Mapping interictal oscillations greater than $200 \mathrm{~Hz}$ recorded with intracranial macroelectrodes in human epilepsy. Brain 2010;133:33-45.

Dümpelmann M, Jacobs J, Kerber K, Schulze-Bonhage A. Automatic 80-250Hz "ripple" high frequency oscillation detection in invasive subdural grid and strip recordings in epilepsy by a radial basis function neural network. Clin Neurophysiol $2012 ; 123: 1721-31$

Fernandez IS, Loddenkemper T. Electrocorticography for seizure foci mapping in epilepsy surgery. J Clin Neurophysiol 2013;30:554-70.

Gardner AB, Worrell GA, Marsh E, Dlugos D, Litt B. Human and automated detection of highfrequency oscillations in clinical intracranial EEG recordings. Clin Neurophysiol 2007;118:1134-43.

Höller Y, Kutil R, Klaffenböck L, Thomschewski A, Höller PM, Bathke AC, et al. High-frequency oscillations in epilepsy and surgical outcome. A meta-analysis. Front Hum Neurosci 2015;9:574. 
Jacobs J, Staba R, Asano E, Otsubo H, Wu JY, Zijlmans M, et al. High-frequency oscillations (HFOs) in clinical epilepsy. Prog Neurobiol. 2012;98:302-15.

Jacobs J, Zijlmans M, Zelmann R, Chatillon CE, Hall J, Olivier A, et al. High-frequency electroencephalographic oscillations correlate with outcome of epilepsy surgery. Ann Neurol 2010;67:209-20.

López-Cuevas A, Castillo-Toledo B, Medina-Ceja L, Ventura-Mejía C, Pardo-Peña K. An algorithm for on-line detection of high frequency oscillations related to epilepsy. Comput Methods Programs Biomed. $2013 ; 110: 354-60$.

Rosso OA, Blanco S, Yordanova J, Kolev V, Figliola A, Schurmann M, et al. Wavelet entropy: a new tool for analysis of short duration brain electrical signals. J Neurosci Methods 2001;105:65-75.

Scheer HJ, Sander T, Trahms L. The influence of amplifier, interface and biological noise on signal quality in high-resolution EEG recordings. Physiol Meas 2006;27:109-17.

Staba RJ, Wilson CL, Bragin A, Fried I, Engel J, Jr. Quantitative analysis of high-frequency oscillations $(80-500 \mathrm{~Hz})$ recorded in human epileptic hippocampus and entorhinal cortex. J Neurophysiol 2002;88:1743-52.

Stockwell RG, Mansinha L, Lowe RP. Localization of the complex spectrum: the S transform. IEEE Trans Sig Proc 1996; 44:998-1001.

Urrestarazu E, Chander R, Dubeau F, Gotman J. Interictal high-frequency oscillations (100-500 Hz) in the intracerebral EEG of epileptic patients. Brain. 2007;130:2354-66.

van 't Klooster MA, Leijten FS, Huiskamp G, Ronner HE, Baayen JC, van Rijen PC, et al. High frequency oscillations in the intra-operative ECoG to guide epilepsy surgery ("The HFO Trial"): study protocol for a randomized controlled trial. Trials 2015a 23;16:422.

van 't Klooster MA, van Klink NE, Leijten FS, Zelmann R, Gebbink TA, Gosselaar PH, et al. Residual fast ripples in the intraoperative corticogram predict epilepsy surgery outcome. Neurology 2015 14;85:120-8.

van Klink NEC, van 't Klooster MA, Zelmann R, Leijten FSS, Ferrier CH, Braun KPJ, et al. High Frequency Oscillations In Intra-Operative Electrocorticography Before And After Epilepsy Surgery. Clin Neurophysiol. 2014 Nov;125(11):2212-9.

von Ellenrieder N, Andrade-Valenca LP, Dubeau F, Gotman J. Automatic detection of fast oscillations (40-200 Hz) in scalp EEG recordings. Clin Neurophysiol 2012;123:670-80.

von Ellenrieder N, Beltrachini L, Perucca P, Gotman J. Size of cortical generators of epileptic interictal events and visibility on scalp EEG. NeuroImage 2014;94:47-54.

Waterstraat G, Fedele T, Burghoff M, Scheer HJ, Curio G. Recording human cortical population spikes non-invasively - An EEG tutorial. J Neurosci Methods 2015;250:74-84.

Worrell GA, Gardner AB, Stead SM, Hu S, Goerss S, Cascino GJ, et al. High-frequency oscillations in human temporal lobe: simultaneous microwire and clinical macroelectrode recordings. Brain 2008;131:928-37. 
Wu JY, Sankar R, Lerner JT, Matsumoto JH, Vinters HV, Mathern GW. Removing interictal fast ripples on electrocorticography linked with seizure freedom in children. Neurology 2010;75:1686-94.

Zelmann R, Mari F, Jacobs J, Zijlmans M, Chander R, Gotman J. Automatic detector of high frequency oscillations for human recordings with macroelectrodes. Conf Proc IEEE Eng Med Biol Soc 2010; 2010:2329-2333.

Zelmann R, Mari F, Jacobs J, Zijlmans M, Dubeau F, Gotman J. A comparison between detectors of high frequency oscillations. Clin Neurophysiol 2012; 123:106-16.

Zijlmans M, Huiskamp GM, Cremer OL, Ferrier CH, van Huffelen AC, Leijten FS. Epileptic highfrequency oscillations in intraoperative electrocorticography: the effect of propofol. Epilepsia 2012a;53:1799-809.

Zijlmans M, Jacobs J, Zelmann R, Dubeau F, Gotman J. High-frequency oscillations mirror disease activity in patients with epilepsy. Neurology 2009;72:979-86.

Zijlmans M, Jiruska P, Zelmann R, Leijten FS, Jefferys JG, Gotman J. High-frequency oscillations as a new biomarker in epilepsy. Ann Neurol 2012;71:169-78.

Zweiphenning W, van 't Klooster M, van Diessen E, van Klink N, Huiskamp G, et al., High frequency oscillations and high frequency functional network characteristics in the intra-operative electrocorticogram in temporal lobe epilepsy. $2^{\text {nd }}$ International workshop on High Frequency Oscillations in Epilepsy, Freiburg 2016. 


\section{Supplementary material}

\section{S1. Optimization procedure on dataset 1}

An extract of the performance of the detector in the calibration stage on dataset 1 is shown is Table S1 for ripples (Table S1a) and FRs (Table S1b). Different rows correspond to different values for the parameters $\mathrm{BL}_{\mathrm{thr}}, \mathrm{CDF}_{\mathrm{thr}}, \mathrm{t}_{\mathrm{THR}}$. The detector performance is evaluated in terms of TPR, FPR and AUC averaged across patients. ROC values reported here describe the performance in the test set.

\begin{tabular}{|c|c|c|c|c|c|}
\hline \multicolumn{7}{|c|}{ (a) ripples } \\
\hline \multicolumn{7}{|c|}{ SETTINGS } & \multicolumn{3}{c|}{ ROC } \\
\hline BLthr & CDF $_{\text {thr }}$ & tTHR $^{\prime}$ & TPR & FPR & AUC \\
\hline 0.85 & 0.99 & $10 \mathrm{~ms}$ & $1.000(0.000)$ & $0.039(0.015)$ & $0.981(0.008)$ \\
\hline 0.95 & 0.99 & $10 \mathrm{~ms}$ & $0.990(0.038)$ & $0.223(0.085)$ & $0.883(0.054)$ \\
\hline 0.85 & 0.99 & $15 \mathrm{~ms}$ & $0.992(0.021)$ & $0.017(0.009)$ & $0.988(0.014)$ \\
\hline 0.95 & 0.99 & $15 \mathrm{~ms}$ & $0.987(0.039)$ & $0.119(0.059)$ & $0.934(0.044)$ \\
\hline $\mathbf{0 . 8 5}$ & $\mathbf{0 . 9 9}$ & $\mathbf{2 0} \mathbf{~ m s}$ & $\mathbf{0 . 9 4 4}(\mathbf{0 . 1 3 5})$ & $\mathbf{0 . 0 0 9}(\mathbf{0 . 0 0 5})$ & $\mathbf{0 . 9 6 8 ( 0 . 0 6 8 )}$ \\
\hline 0.95 & 0.99 & $20 \mathrm{~ms}$ & $0.980(0.048)$ & $0.068(0.041)$ & $0.956(0.042)$ \\
\hline
\end{tabular}

\begin{tabular}{|c|c|c|c|c|c|}
\hline \multicolumn{7}{|c|}{ (b) FRs } \\
\hline \multicolumn{7}{|c|}{ SETTINGS } & \multicolumn{3}{c|}{ ROC } \\
\hline BL $_{\text {thr }}$ & CDF $_{\text {thr }}$ & t $_{\text {THR }}$ & TPR & FPR & AUC \\
\hline 0.85 & 0.99 & $5 \mathrm{~ms}$ & $0.920(0.181)$ & $0.0025(0.0035)$ & $0.959(0.091)$ \\
\hline 0.95 & 0.99 & $5 \mathrm{~ms}$ & $0.930(0.164)$ & $0.0082(0.0153)$ & $0.961(0.086)$ \\
\hline $\mathbf{0 . 8 5}$ & $\mathbf{0 . 9 9}$ & $\mathbf{1 0} \mathbf{~ m s}$ & $\mathbf{0 . 8 2 3}(\mathbf{0 . 3 5 8})$ & $\mathbf{0 . 0 0 0 2 ( 0 . 0 0 0 5 )}$ & $\mathbf{0 . 9 1 1 ( 0 . 1 7 9 )}$ \\
\hline 0.95 & 0.99 & $10 \mathrm{~ms}$ & $0.832(0.345)$ & $0.0008(0.0021)$ & $0.916(0.173)$ \\
\hline 0.85 & 0.99 & $15 \mathrm{~ms}$ & $0.620(0.472)$ & $0.0001(0.0004)$ & $0.810(0.236)$ \\
\hline 0.95 & 0.99 & $15 \mathrm{~ms}$ & $0.623(0.469)$ & $0.0004(0.0012)$ & $0.812(0.235)$ \\
\hline
\end{tabular}

Table S1. ROC analysis on dataset 1 for different input parameters settings. (a) Ripples had comparable TPR and AUC for all settings, while FPR was very low for $\mathrm{BL}_{\text {thr }}=0.85, \mathrm{CDF}_{\text {thr }}=$ 0.99, $\mathrm{tTHR}_{\mathrm{THR}}=20 \mathrm{~ms}$. (b) FRs had comparable TPR and AUC for tTHR $=5 \mathrm{~ms}$ and $\mathrm{t}_{\mathrm{THR}}=10 \mathrm{~ms}$, while FPR was very low for $\mathrm{BL}_{\mathrm{thr}}=0.85, \mathrm{CDF}_{\mathrm{thr}}=0.99, \mathrm{t}_{\mathrm{THR}}=10 \mathrm{~ms} . \mathrm{TPR}, \mathrm{FPR}, \mathrm{AUC}$ are reported as mean(std) across patients. Selected optimal settings are in bold face. 


\section{S2. FR rate for each patient}

FR rates before and after thresholding (FR rate per single channel $>1$ ) are reported in Table S2.

\begin{tabular}{|c|c|c|c|c|c|c|c|}
\hline \multirow{2}{*}{ patient } & \multirow{2}{*}{ outcome } & \multicolumn{2}{|c|}{ Visual } & \multicolumn{2}{|c|}{ Automatic } & \multicolumn{2}{|c|}{$\begin{array}{c}\text { Automatic } \\
\text { Thresholded }\end{array}$} \\
\hline & & events & $\begin{array}{c}\text { mean } \\
\text { rate }\end{array}$ & events & $\begin{array}{c}\text { mean } \\
\text { rate }\end{array}$ & events & $\begin{array}{c}\text { mean } \\
\text { rate }\end{array}$ \\
\hline 1 & 1 & 0 & 0 & 0 & 0 & 0 & 0 \\
\hline 2 & 1 & 0 & 0 & 0 & 0 & 0 & 0 \\
\hline 3 & 1 & 0 & 0 & 2.0 & 1.0 & 0 & 0 \\
\hline 4 & 1 & 0 & 0 & 0 & 0 & 0 & 0 \\
\hline 5 & 1 & 0 & 0 & 0 & 0 & 0 & 0 \\
\hline 6 & 1 & 0 & 0 & 0 & 0 & 0 & 0 \\
\hline 7 & 0 & 3.0 & 1.5 & 0 & 0 & 0 & 0 \\
\hline 8 & 1 & 3.0 & 3.0 & 0 & 0 & 0 & 0 \\
\hline 9 & 1 & 0 & 0 & 0 & 0 & 0 & 0 \\
\hline 10 & 0 & 0 & 0 & 0 & 0 & 0 & 0 \\
\hline 11 & 0 & 0 & 0 & 0 & 0 & 0 & 0 \\
\hline 12 & 1 & 0 & 0 & 0 & 0 & 0 & 0 \\
\hline 13 & 1 & 30.0 & 2.7 & 1.0 & 1.0 & 0 & 0 \\
\hline 14 & 0 & 0 & 0 & 2.0 & 1.0 & 0 & 0 \\
\hline 15 & 0 & 0 & 0 & 0 & 0 & 0 & 0 \\
\hline 16 & 0 & 0 & 0 & 0 & 0 & 0 & 0 \\
\hline 17 & 1 & 0 & 0 & 0 & 0 & 0 & 0 \\
\hline 18 & 0 & 0 & 0 & 0 & 0 & 0 & 0 \\
\hline 19 & 0 & 0 & 0 & 0 & 0 & 0 & 0 \\
\hline 20 & 1 & 0 & 0 & 0 & 0 & 0 & 0 \\
\hline 21 & 0 & 0 & 0 & 0 & 0 & 0 & 0 \\
\hline 22 & 1 & 0 & 0 & 1.0 & 1.0 & 0 & 0 \\
\hline 23 & 0 & 0 & 0 & 25.0 & 2.8 & 23.0 & 3.3 \\
\hline 24 & 1 & 4.0 & 1.3 & 0 & 0 & 0 & 0 \\
\hline 25 & 1 & 0 & 0 & 0 & 0 & 0 & 0 \\
\hline 26 & 1 & 0 & 0 & 0 & 0 & 0 & 0 \\
\hline 27 & 0 & 1.0 & 1.0 & 0 & 0 & 0 & 0 \\
\hline 28 & 1 & 0 & 0 & 1.0 & 1.0 & 0 & 0 \\
\hline 29 & 0 & 0 & 0 & 0 & 0 & 0 & 0 \\
\hline 30 & 1 & 0 & 0 & 0 & 0 & 0 & 0 \\
\hline 31 & 1 & 0 & 0 & 3.0 & 1.0 & 0 & 0 \\
\hline 32 & 0 & 0 & 0 & 0 & 0 & 0 & 0 \\
\hline 33 & 0 & $\begin{array}{l}50.0 \\
\end{array}$ & 25.0 & 5.0 & 5.0 & 5.0 & 5.0 \\
\hline 34 & 0 & 0 & 0 & 1.0 & 1.0 & 0 & 0 \\
\hline 35 & 0 & 0 & 0 & 0 & 0 & 0 & 0 \\
\hline 36 & 0 & 0 & 0 & 0 & 0 & 0 & 0 \\
\hline 37 & 1 & 0 & 0 & 0 & 0 & 0 & 0 \\
\hline 38 & 0 & 62.0 & 8.9 & 17.0 & 2.4 & 13.0 & 4.3 \\
\hline 39 & 1 & 0 & 0 & 1.0 & 1.0 & 0 & 0 \\
\hline 40 & 0 & 0 & 0 & 0 & 0 & 0 & 0 \\
\hline 41 & 0 & 2.0 & 1.0 & 2.0 & 1.0 & 0 & 0 \\
\hline 42 & 1 & 0 & 0 & 1.0 & 1.0 & 0 & 0 \\
\hline 43 & 1 & 0 & 0 & 0 & 0 & 0 & 0 \\
\hline
\end{tabular}




\begin{tabular}{|l|c|c|c|c|c|c|c|}
\hline 44 & 0 & 2.0 & 1.0 & 2.0 & 1.0 & 0 & 0 \\
\hline 45 & 1 & 0 & 0 & 0 & 0 & 0 & 0 \\
\hline 46 & 1 & 0 & 0 & 1.0 & 1.0 & 0 & 0 \\
\hline 47 & 1 & 0 & 0 & 0 & 0 & 0 & 0 \\
\hline 48 & 1 & 0 & 0 & 0 & 0 & 0 & 0 \\
\hline 49 & 1 & 0 & 0 & 0 & 0 & 0 & 0 \\
\hline 50 & 0 & 42.0 & 3.0 & 6.0 & 1.5 & 4.0 & 2.0 \\
\hline 51 & 1 & 0 & 0 & 0 & 0 & 0 & 0 \\
\hline 52 & 1 & 0 & 0 & 1.0 & 1.0 & 0 & 0 \\
\hline 53 & 0 & 202.0 & 50.5 & 10.0 & 5.0 & 10.0 & 5.0 \\
\hline 54 & 0 & 10.0 & 2.5 & 13.0 & 3.3 & 12.0 & 4.0 \\
\hline
\end{tabular}

Table S2. FRs rate in dataset 2. In column-wise order: Patient number, Outcome ( $1=$ good, 0 = poor), visual marking total rate, visual marking mean rate (computed over channel with events), automatic detection total rate, automatic detection mean rate (computed over channel with events), automatic detection total rate with threshold, automatic detection mean rate (computed over channel with events) with threshold. The threshold was set at $>1$ FR per channel. Good outcome and HFO rates in patients with poor outcome are highlighted in green. HFO rates in patients with good outcome are highlighted in red. 


\section{Figure legends}

Figure 1. Example of automatically detected HFO. (A) Raw ECoG. (B) Ripple Band (80-250 $\mathrm{Hz}$ ) filtered signal with envelope (red). (C) Fast ripples (FR) Band (250-500 Hz) filtered signal. The threshold computed in stage I is displayed as a black horizontal line. (D) Time frequency representation. The ripple and the FR stand out in the time-frequency plane as two separate blobs. (E) Instantaneous Power Spectrum averaged across the EoI (white lines in panel D) shows spectral peak in the ripple range and the FR range.

Figure 2. Example of HFO detector performance in the ripple band mapped in the ROC plane. The three points and their inlays in the ROC plane display the detection of ripples following different combinations of the three input parameters for one representative patient from the calibration dataset. Automatically detected ripples are highlighted in green and visually marked ripples are highlighted in red. The higher true positive rate (TPR) implies the presence of a higher number of false positives (green markings without underlying red bar) (C), while the lower TPR entailed increasing false negatives (red markings not overlapped by green ones) (A). The situation in the middle (B) describes the target of our analysis: a trade-off between these two extremes ensures a reliable detection, favoring high specificity at the expense of sensitivity.

Figure 3. Example of a patient with refractory focal epilepsy that underwent resective surgery of a dysembryoplastic neuroepithelial tumor in the left central region. (A) Pre-resection photograph. (B) Post-resection photograph with schematic of the ECoG placements. Fast ripples (FRs) in post-resection $\mathrm{ECoG}$ correspond with recurrent auras after surgery (Engel 1B). HFOs are represented in the bipolar channel montage of the two performed postresection ECoG. The resected area is delineated by a dotted white line. Note that FRs (yellow) were present in the margin of the resected lesion, ripples (green) in a larger region. (C) ECoG signal in raw, ripple $(80-250 \mathrm{~Hz})$ and FR frequency $(250-500 \mathrm{~Hz})$ band in a selection of four bipolar channels. What can be seen are spikes (in the raw signal), ripples and FR in channels 2 and 3. (D) Event rates for ripples and FRs from visual marking (blue) and automatic detection (brown).

Figure 4. FRs with visual marking and automatic detection. Raw data, FR range band-passed data and the Stockwell transform are shown for an interval of $200 \mathrm{~ms}$ around a FR event. The 
blue bar in the FR band indicates the event duration (A) True positive (TP): FR identified by both visual marking and automatic detection in a patient with poor outcome (patient 53). (B) False positive (FP): FR identified by automatic detection but not by visual marking in a patient with poor outcome (patient 7). (C) False negative (FN): FR marked visually but not detected automatically in a patient with good outcome (patient 8).

Figure 5. Distribution of the mean event rate (events/min/channels with events) per patient of post-resection fast ripples (FRs) for good and poor outcome. (A) Automatically detected FRs occurred only in patients with poor outcome (Mann-Whitney $U, p=0.004$, patients without events are included). (B) Visually marked FRs occurred in patients with good and with poor outcome, $(\mathrm{p}=0.018$, Mann-Whitney U, patients without events included) (Data from van 't Klooster et al., 2015b).

\section{Tables}

\begin{tabular}{|c|c|c|}
\hline $\mathrm{TP}$ & true positive & Event that is automatically detected and visually marked \\
\hline $\mathrm{TN}$ & true negative & Correctly detected baseline interval \\
\hline FP & false positive & $\begin{array}{r}\text { Event that is automatically detected but NOT visually } \\
\text { marked }\end{array}$ \\
\hline FN & false negative & $\begin{array}{r}\text { Event that is NOT automatically detected but is visually } \\
\text { marked }\end{array}$ \\
\hline TPR & true positive rate & $\mathrm{TP} /(\mathrm{TP}+\mathrm{FN})$ \\
\hline FPR & false positive rate & $\mathrm{FP} /(\mathrm{FP}+\mathrm{TN})$ \\
\hline AUC & $\begin{array}{l}\text { area under curve } \\
\text { (cost function) }\end{array}$ & $0.5+0.5 * \mathrm{TPR}+0.5 * \mathrm{FPR}$ \\
\hline Sensitivity & & TPR \\
\hline Specificity & & $1-\mathrm{FPR}$ \\
\hline
\end{tabular}

Table 1. Elements of the Receiver Operating Characteristics (ROC). 


\begin{tabular}{|c|c|}
\hline PPV & $\frac{\text { patients with recurrent seizures and residual ECoG events }}{\text { patients with automatically detected events }}$ \\
\hline$N P V$ & $\frac{\text { patients with seizure freedom and no residual ECoG events }}{\text { patients without residual events }}$ \\
\hline
\end{tabular}

Table 2. Definition of positive predictive value (PPV) and negative predictive value (NPV). 

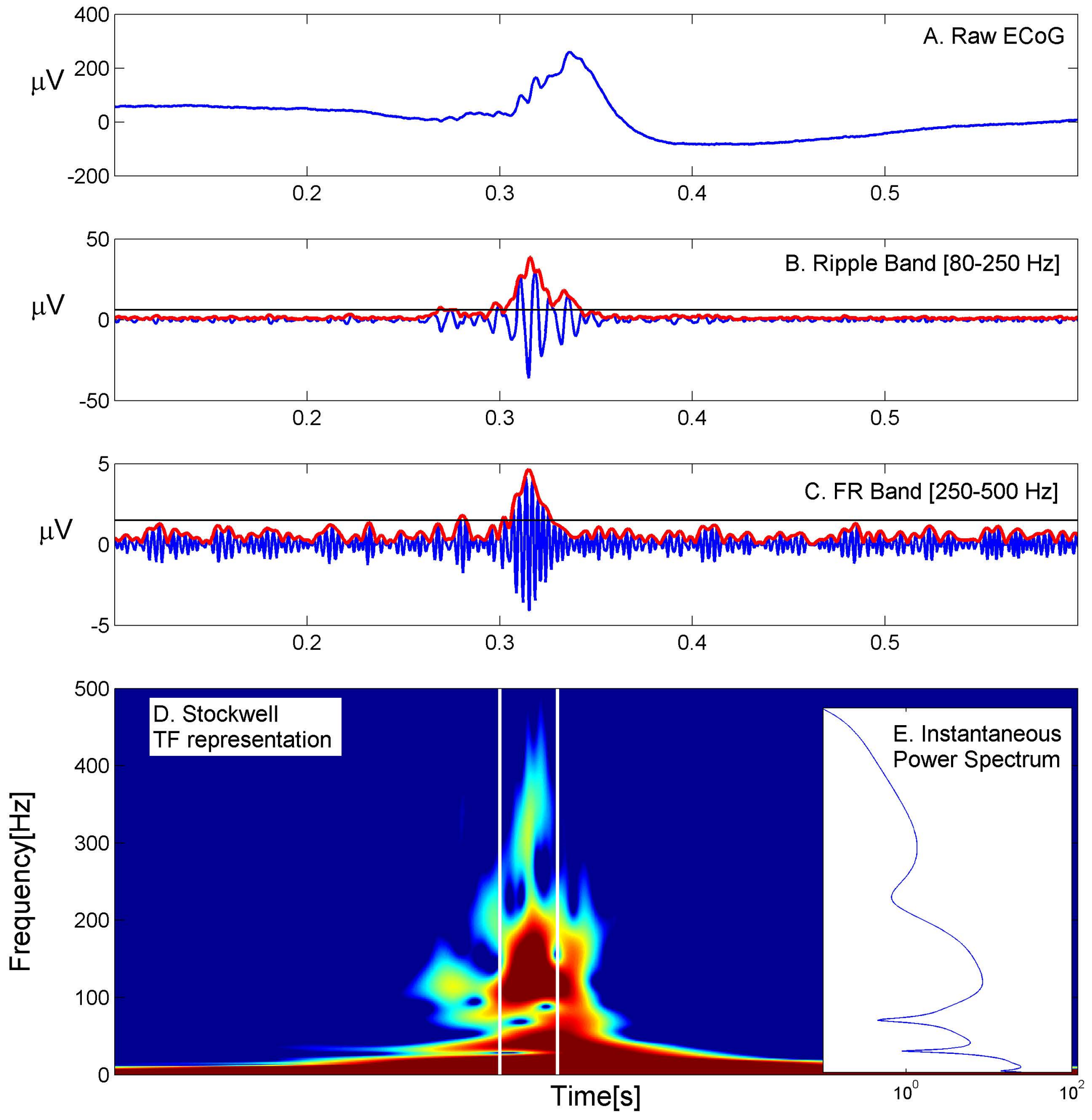


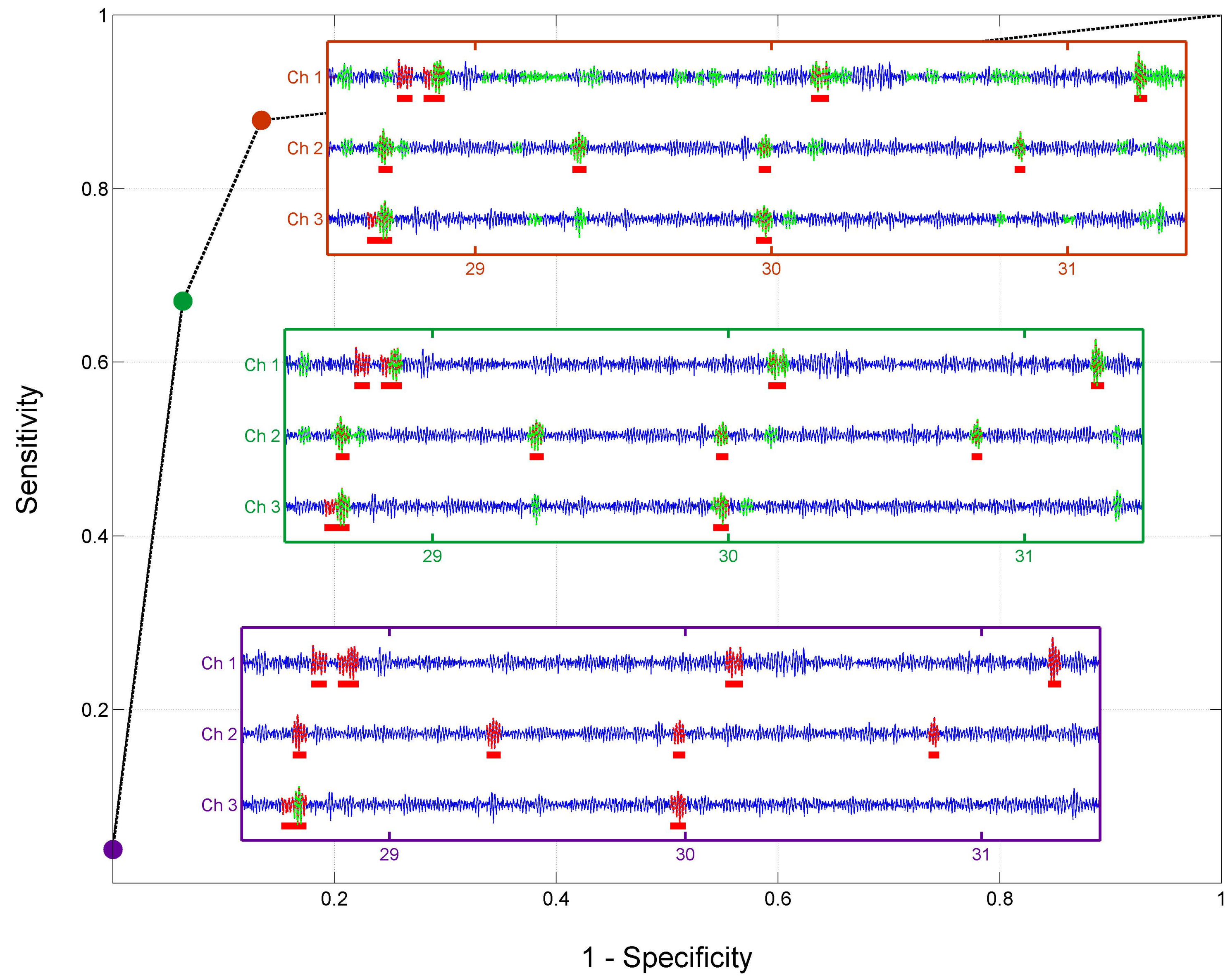




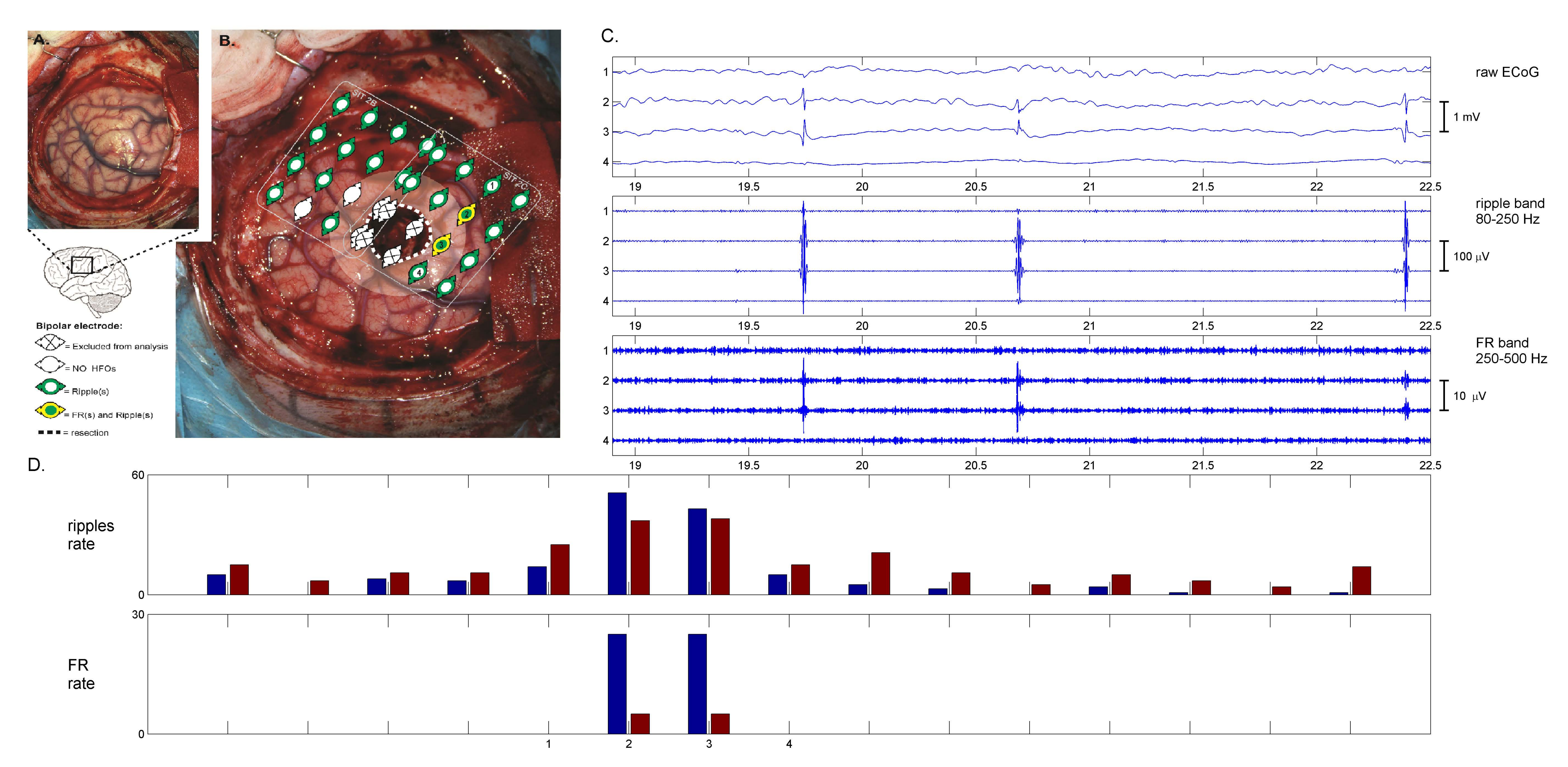


A. True Positive
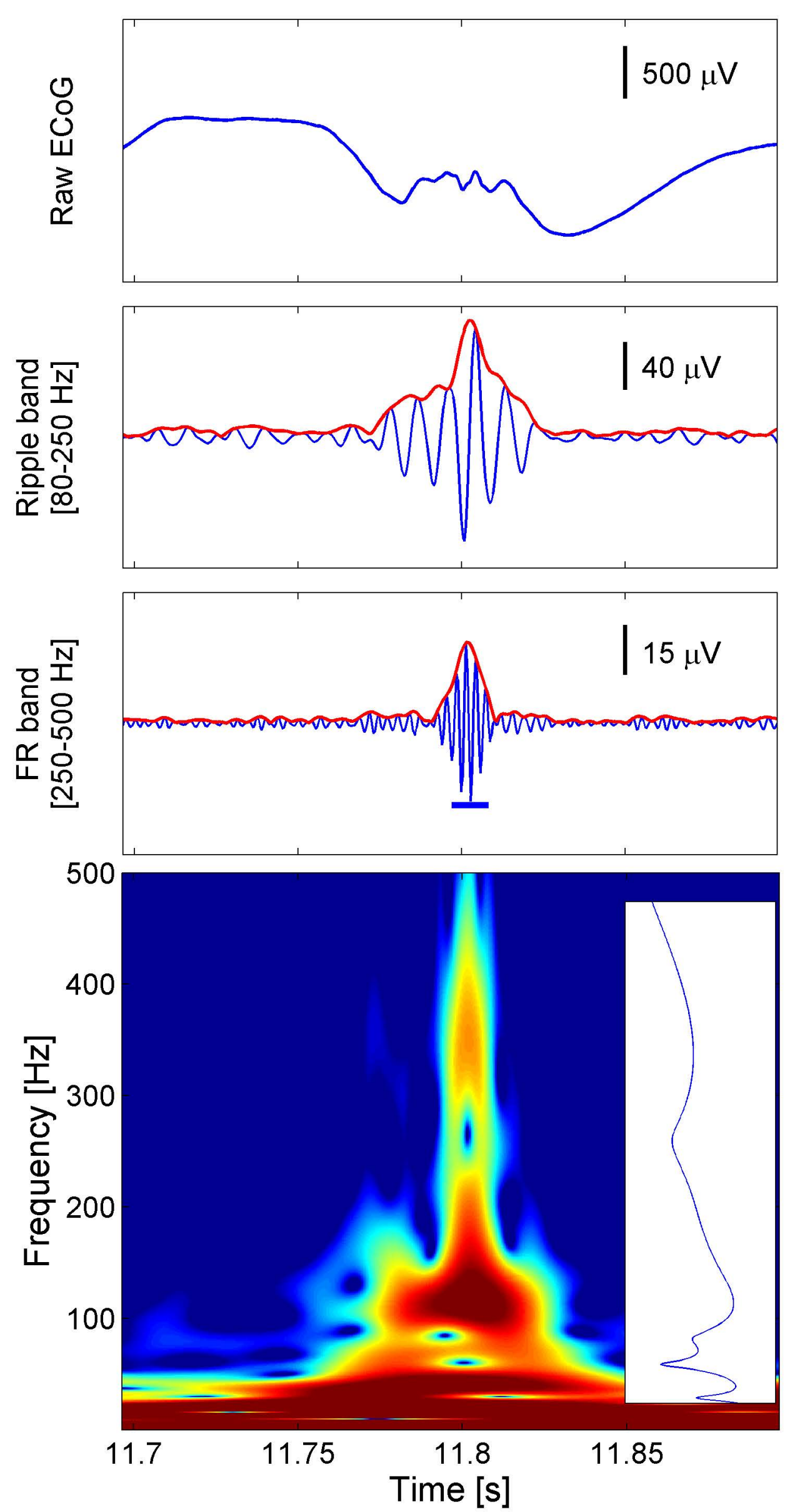
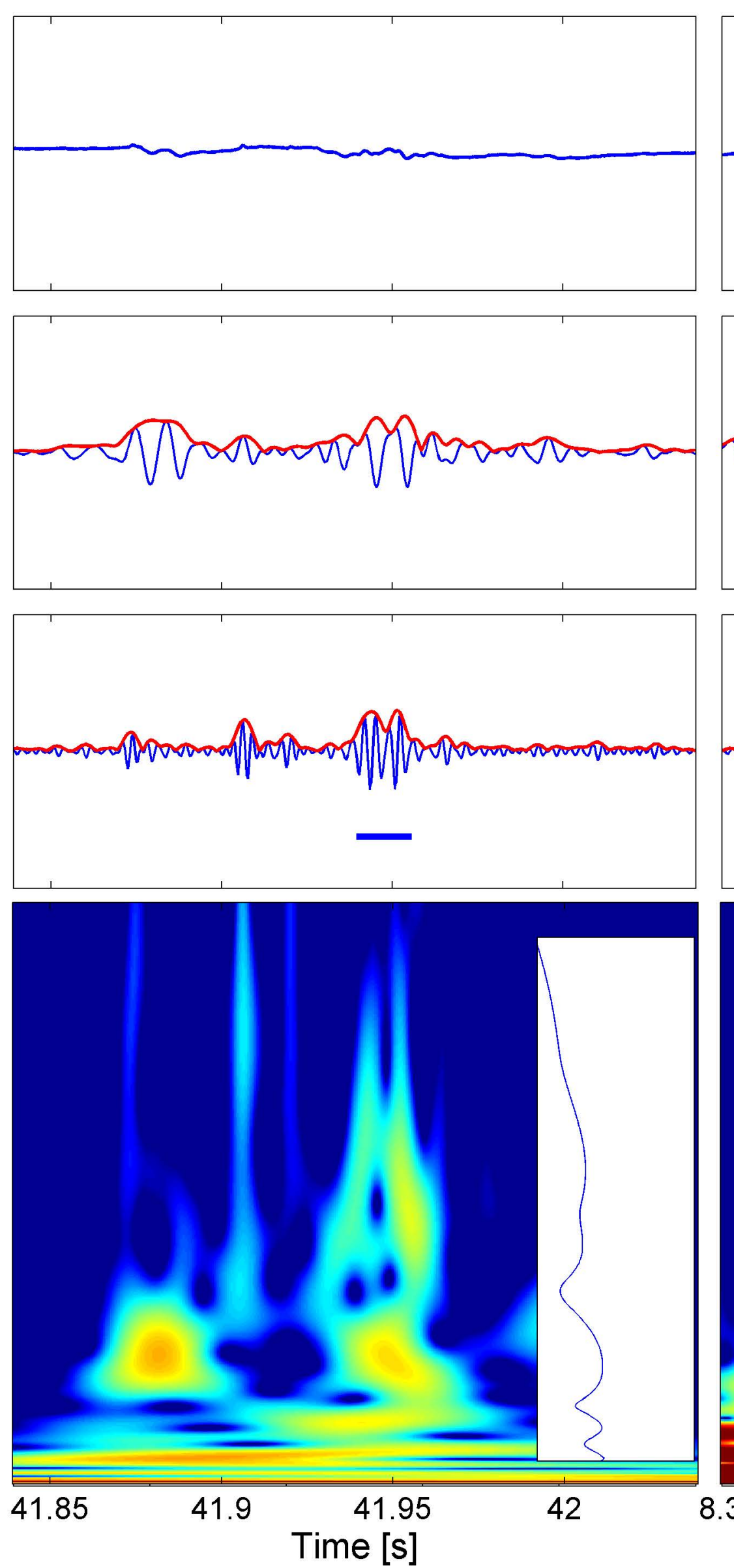
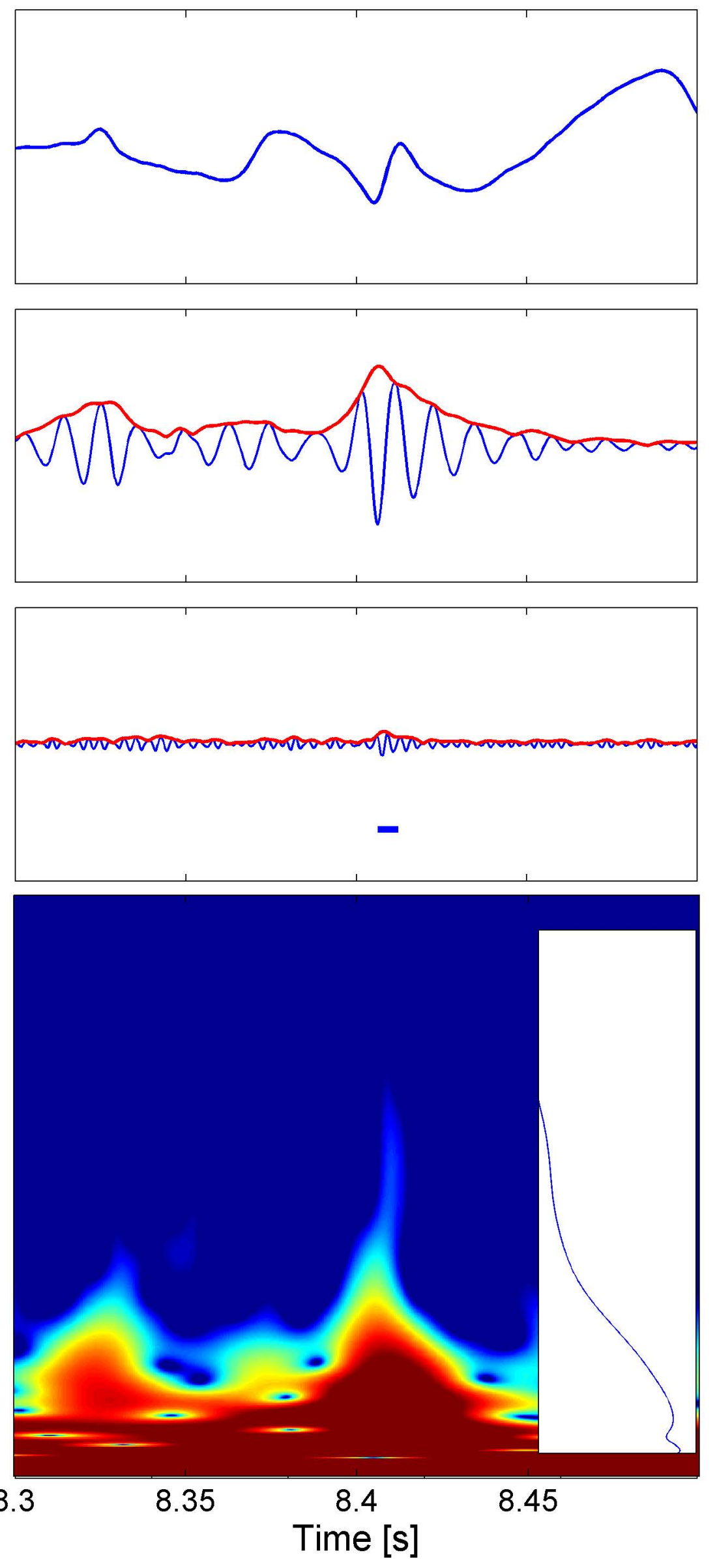

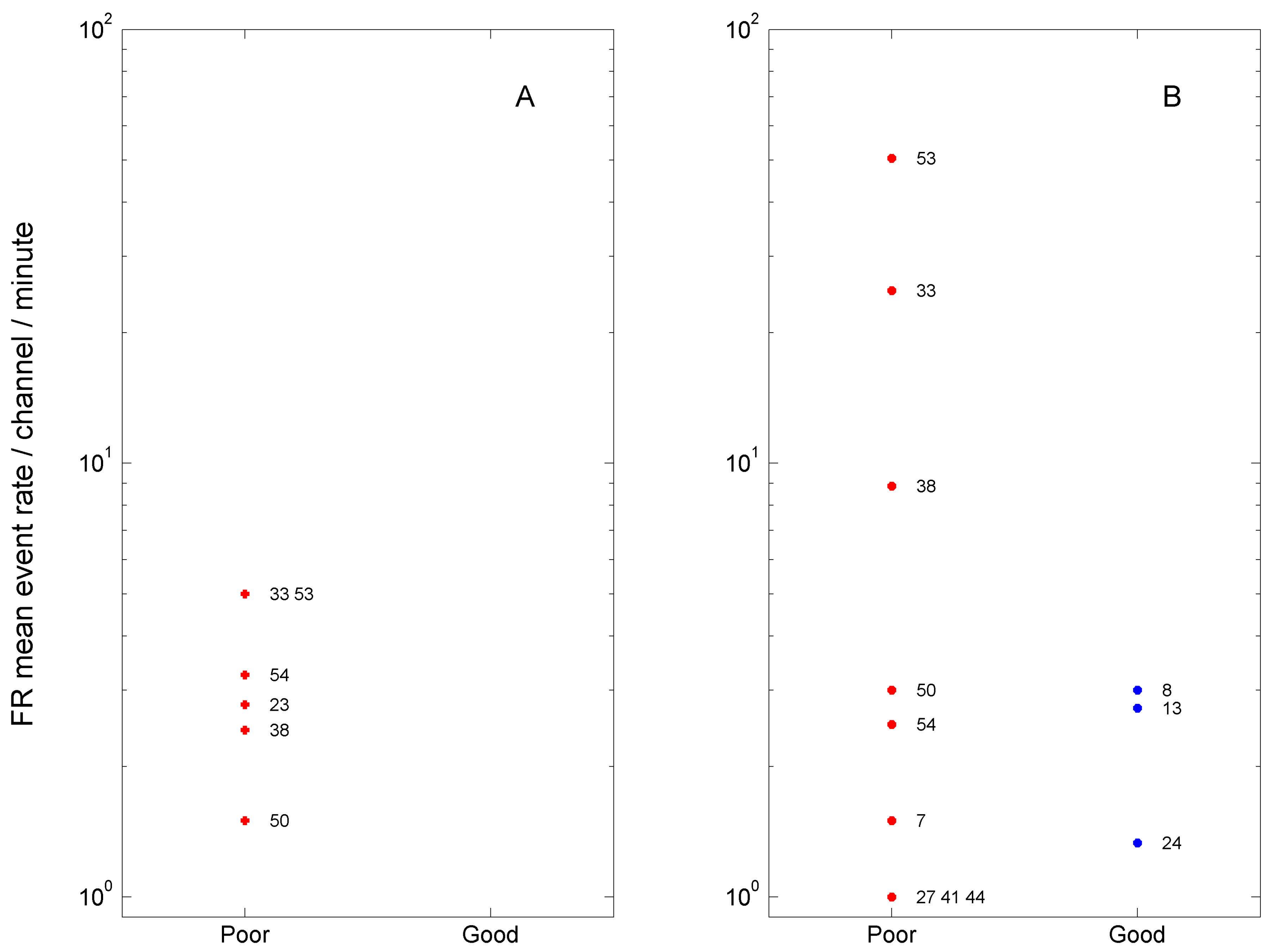